\title{
A Verification-driven Approach to Control Analysis and Tuning
}

\author{
Luis G. Crespo* \\ National Institute of Aerospace \\ Sean P. Kenny and Daniel P. Giesy ${ }^{\dagger}$ \\ Dynamic Systems and Control Branch, NASA Langley Research Center
}

\begin{abstract}
This paper proposes a methodology for the analysis and tuning of controllers using control verification metrics. These metrics, which are introduced in a companion paper, measure the size of the largest uncertainty set of a given class for which the closed-loop specifications are satisfied. This framework integrates deterministic and probabilistic uncertainty models into a setting that enables the deformation of sets in the parameter space, the control design space, and in the union of these two spaces. In regard to control analysis, we propose strategies that enable bounding regions of the design space where the specifications are satisfied by all the closed-loop systems associated with a prescribed uncertainty set. When this is unfeasible, we bound regions where the probability of satisfying the requirements exceeds a prescribed value. In regard to control tuning, we propose strategies for the improvement of the robust characteristics of a baseline controller. Some of these strategies use multi-point approximations to the control verification metrics in order to alleviate the numerical burden of solving a min-max problem. Since this methodology targets non-linear systems having an arbitrary, possibly implicit, functional dependency on the uncertain parameters and for which high-fidelity simulations are available, they are applicable to realistic engineering problems.
\end{abstract}

Keywords: control verification, uncertainty, robustness, control tuning.

\section{Acronyms}

CDV : Critical Design Value

CPV : Critical Parameter Value

CSR : Critical Similitude Ratio

MS : Maximal Set

PSM : Parametric Safety Margin

RI : Reliability Index

\section{Introduction}

Over the last two decades there has been a flurry of research concentrating on robust stability to real uncertain parameters. Suffice it is to say there are now a number of results applicable to linear

${ }^{*}$ Senior Staff Scientist, 100 Exploration Way, Hampton VA 23666 USA. AIAA Professional Member.

${ }^{\dagger}$ Aerospace Technologists, Mail Stop 308, NASA LaRC, Hampton VA 23681 USA. 
systems having linear uncertainty structures (see [1-7] and their bibliographies) and polynomial parameter dependencies (see [5-7]). At the control verification stage, we usually have a highfidelity dynamic model for which a deterministic or probabilistic uncertainty model is available, and where stability and performance specifications are both present. This commonly entails a nonlinear closed-loop system where the functional relationship between the design specifications and the uncertainty is arbitrary and may only be known implicitly, e.g., the dependence of the time response of a nonlinear system on the initial condition. Under these conditions, the vast majority of assumptions behind robust and adaptive control methods (e.g., linear dynamics, multi-affine parameter dependencies, existence of matching conditions) are inapplicable, unverifiable, or require over-bounding. Even though such assumptions enable a mathematically rigorous manipulation of the problem, only the system's physics will validate the effectiveness of the resulting controllers.

The methodology proposed herein will not force the physics to fit into a conveniently posed mathematical framework, but it will develop mathematics that enable the analysis and tuning of controllers according to their performance in dynamic models having varying levels of fidelity. This implies that the structure of the plant is arbitrary and a baseline controller, possibly designed using a simpler dynamic model and carrying along a set of assumptions we do not need/want to know about, is available. Few methods in the literature ${ }^{8-11}$ deal with systems of this complexity, those based on Monte Carlo analysis being the most widely used. ${ }^{12,13}$ In regard to control analysis, we propose strategies that enable bounding regions of the design space (i.e., the space of control gains for a fixed control structure) where the specifications are satisfied by all the closed-loop systems associated with a prescribed uncertainty set. When this is unfeasible, we bound regions where the probability of satisfying the specifications exceeds a prescribed value. The search for robustly optimal controllers can be efficiently made by constraining the admissible design space to these bounding sets. In regard to control tuning, we propose strategies that improve the robust characteristics of a baseline controller by maximizing the control verification metrics proposed in Reference [14]. Formulations that alleviate the numerical burden of solving a min-max problem are also proposed.

This paper is organized as follows. Section II introduces the notions and formulations required to deform sets in the parameter space. This is followed by Section III, where extensions that allow for the exploration of the design space are considered. Section IV presents formulations that enable the search for robustly optimal controllers, including some that relax the numerical demands of the search. As an example, a baseline controller originally designed for the robust control challenge problem posed in the 1990 American Control Conference is tuned. Finally, a few concluding remarks close the paper.

\section{Background}

This section presents a summary of the developments in Reference [14] that are essential to this paper. Interested readers should resort to this reference for additional details.

\section{II.A. Concepts and Notions}

The concern in this paper is the analysis and tuning of controlled systems having a parametric mathematical model. The parameters which specify the closed-loop system are grouped into two categories: uncertain parameters, which are denoted by the vector $\boldsymbol{p}$, and the control design parameters, which are denoted by the vector $\boldsymbol{d}$. While the plant model depends on $\boldsymbol{p}$, the controller depends on $\boldsymbol{d}$.

The uncertainty model of $\boldsymbol{p}$ can be deterministic or probabilistic. A deterministic uncertainty

model is prescribed by the Uncertainty Set $\Delta$, while a probabilistic one is prescribed by a random 
vector. The distribution of this vector is specified by the joint probability density function $f_{\boldsymbol{p}}(\boldsymbol{p})$ defined over $\Delta$. The uncertainty set of the probabilistic model is commonly called the Support Set. Hereafter, the terms uncertainty set and support set will be used interchangeably. Any member of the uncertainty set is called a Realization. The Nominal Parameter value, denoted as $\overline{\boldsymbol{p}}$, is a parameter realization regarded as a good deterministic representation of $\boldsymbol{p}$. Additionally, we will call the set of control design parameters of a baseline controller the Nominal Design point, $\overline{\boldsymbol{d}}$.

Stability and performance requirements for the closed-loop system will be prescribed by the set of constraint functions, $\boldsymbol{g}(\boldsymbol{p}, \boldsymbol{d})<\mathbf{0}$, which depend on the uncertain and control parameters. Throughout this paper, it is assumed that vector inequalities hold component wise. The controller associated with $\overline{\boldsymbol{d}}$ is deemed acceptable if the constraints are satisfied for enough, if not all, parameter realizations.

Sets in the parameter and design spaces, instrumental to the developments that follow are introduced next. The Failure Domain is given by ${ }^{\mathrm{a}}$

$$
\begin{gathered}
\mathcal{F}_{\boldsymbol{p}, \boldsymbol{d}}^{j} \triangleq\left\{\langle\boldsymbol{p}, \boldsymbol{d}\rangle: \boldsymbol{g}_{j}(\boldsymbol{p}, \boldsymbol{d}) \geq 0\right\}, \\
\mathcal{F}_{\boldsymbol{p}, \boldsymbol{d}} \triangleq \bigcup_{j=1}^{\operatorname{dim}(\boldsymbol{g})} \mathcal{F}_{\boldsymbol{p}, \boldsymbol{d}}^{j} .
\end{gathered}
$$

While Equation (1) describes the failure domain corresponding to the $j$ th requirement, Equation (2) describes the failure domain for all requirements. The Non-Failure Domain is the complement set of the failure domain and will be denoted ${ }^{b}$ as $\mathcal{F}^{c}$. The names "failure domain" and "nonfailure domain" are used because in the failure domain at least one constraint is violated while, in the non-failure domain, all constraints are satisfied. The solution set of equation $\max _{j}\left\{\boldsymbol{g}_{j}\right\}=0$ usually partitions the space into these two domains. The projection of the failure domain onto the parameter space when the design point $\boldsymbol{d}$ is kept at its nominal value $\overline{\boldsymbol{d}}$, is given by

$$
\mathcal{F}_{\boldsymbol{p}}(\overline{\boldsymbol{d}}) \triangleq\left\{\boldsymbol{p}:\langle\boldsymbol{p}, \overline{\boldsymbol{d}}\rangle \in \mathcal{F}_{\boldsymbol{p}, \boldsymbol{d}}\right\}
$$

Likewise, if the parameter point $\boldsymbol{p}$ is kept at its nominal value $\overline{\boldsymbol{p}}$, the projection of the failure domain onto the design space is given by

$$
\mathcal{F}_{\boldsymbol{d}}(\overline{\boldsymbol{p}}) \triangleq\left\{\boldsymbol{d}:\langle\overline{\boldsymbol{p}}, \boldsymbol{d}\rangle \in \mathcal{F}_{\boldsymbol{p}, \boldsymbol{d}}\right\} .
$$

Expressions corresponding to a particular requirement result from using $\mathcal{F}$ instead of $\mathcal{F}$ in these two equations. The Feasible Design Space, $\mathcal{E}$, the Robust Design Space, $\mathcal{Q}$, and the $(1-\epsilon)$-Probable Design Space, $\mathcal{D}$, are given by

$$
\begin{aligned}
\mathcal{E}(\overline{\boldsymbol{p}}) & \triangleq\{\boldsymbol{d}: \boldsymbol{g}(\overline{\boldsymbol{p}}, \boldsymbol{d})<\mathbf{0}\}, \\
\mathcal{Q}(\Delta) & \triangleq\{\boldsymbol{d}: \boldsymbol{g}(\boldsymbol{p}, \boldsymbol{d})<\mathbf{0}, \forall \boldsymbol{p} \in \Delta\}, \\
\mathcal{D}\left(f_{\boldsymbol{p}}, \epsilon\right) & \triangleq\{\boldsymbol{d}: P[\boldsymbol{g}(\boldsymbol{p}, \boldsymbol{d}) \geq \mathbf{0}] \leq \epsilon\},
\end{aligned}
$$

where $P[\cdot]$ is the probability operator based on the density function $f_{\boldsymbol{p}}$ and $\epsilon \in[0,1]$. Figure 1 illustrates relevant spaces in a two dimensional setting. Note that $\mathcal{Q} \subset \mathcal{E}$ when $\bar{p} \in \Delta$, and $\mathcal{Q} \subset \mathcal{D}\left(f_{\boldsymbol{p}}, \epsilon_{1}\right) \subset \mathcal{D}\left(f_{\boldsymbol{p}}, \epsilon_{2}\right)$ for all $0<\epsilon_{1}<\epsilon_{2} \leq 1$. Additionally, we have $\mathcal{E}^{c}=\mathcal{F}_{\boldsymbol{d}}(\overline{\boldsymbol{p}}), \mathcal{D}\left(f_{\boldsymbol{p}}, 0\right)=\mathcal{Q}$ and $\mathcal{D}\left(f_{\boldsymbol{p}}, 1\right)=\mathbb{R}^{\operatorname{dim}(\boldsymbol{d})}$. The controller with gains $\overline{\boldsymbol{d}}$ will be called Robust if $\mathcal{F}_{\boldsymbol{p}}(\overline{\boldsymbol{d}})$ and $\Delta$ do not overlap. In such a case, $\overline{\boldsymbol{d}}$ belongs to the robust design space. Otherwise, the controller will be called Non-robust. The level of robustness of a controller is related to the size and geometry of its corresponding non-failure domain.

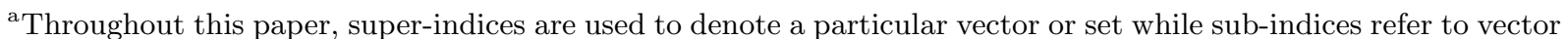
components, e.g., $\boldsymbol{p}_{i}^{j}$ is the $i$ th component of the vector $\boldsymbol{p}^{j}$.

${ }^{\mathrm{b}}$ The complement set operator will be denoted as the super-index $c$.
} 


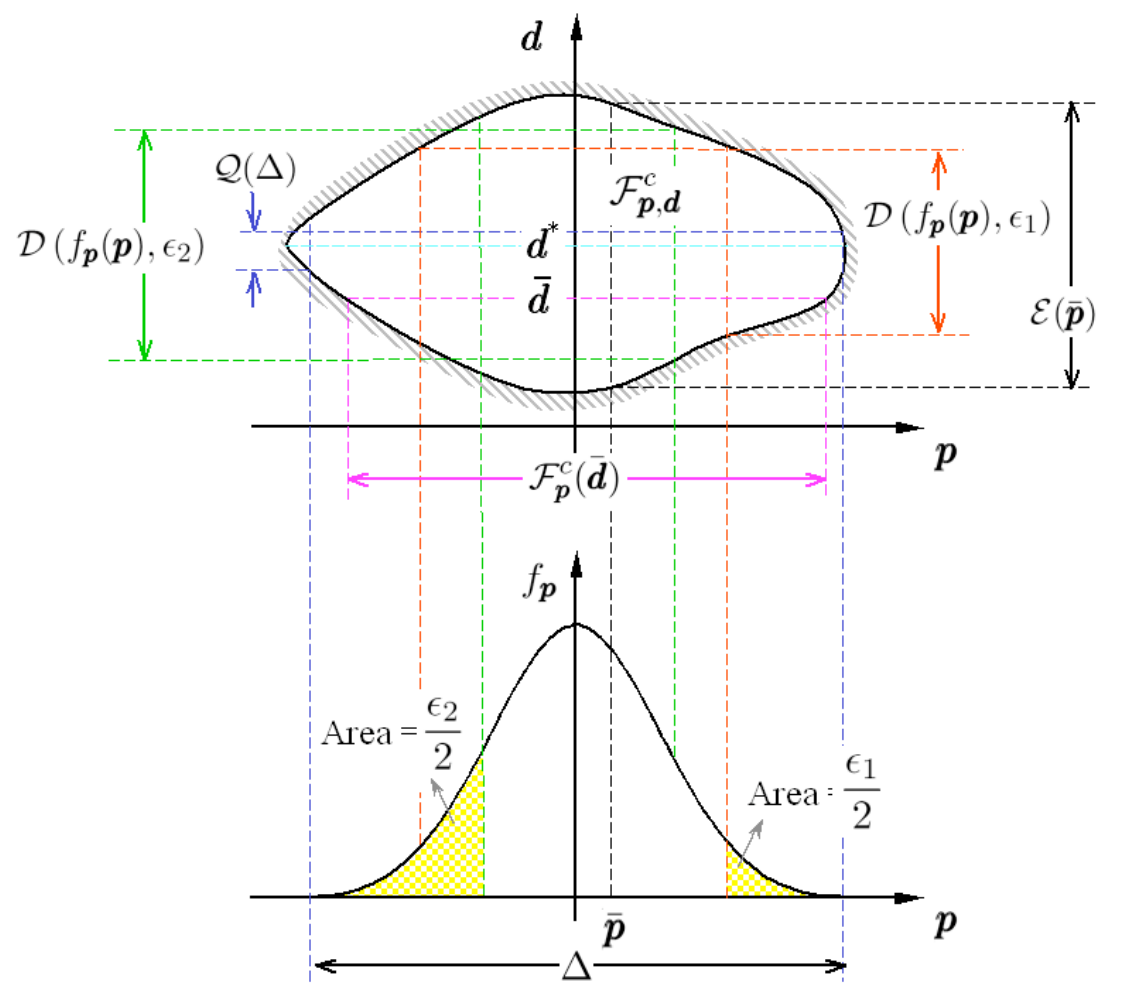

Figure 1. Relevant parameter- and design-spaces.

\section{II.B. Set Deformations}

The mathematical background for deforming sets in the parameter space is presented herein. In this section, the design point will be kept fixed at its nominal value $\overline{\boldsymbol{d}}$, in which case the relevant failure domains are $\mathcal{F}_{\boldsymbol{p}}(\overline{\boldsymbol{d}})$ and $\mathcal{F}_{\boldsymbol{p}}^{j}(\overline{\boldsymbol{d}})$. For simplicity in the notation, we will denote these sets $\mathcal{F}$ and $\mathcal{F}^{j}$.

Let $\Omega$ be a set, called the Reference Set, whose geometric center is the nominal parameter $\overline{\boldsymbol{p}}$. The geometry of this set will be prescribed according to the levels of uncertainty in $\boldsymbol{p}$. One possible choice for the reference set is a hyper-sphere. The hyper-sphere of radius $R$ centered at $\overline{\boldsymbol{p}}$, denoted as $\mathcal{S}(\overline{\boldsymbol{p}}, R)$, is defined by

$$
\mathcal{S}(\overline{\boldsymbol{p}}, R)=\{\boldsymbol{p}:\|\overline{\boldsymbol{p}}-\boldsymbol{p}\| \leq R\} .
$$

Another choice might be to confine each component of the reference set to a bounded interval. This leads to a hyper-rectangular set. If $\boldsymbol{m}>\mathbf{0}$ is the vector of half-lengths of the sides of such a set, the hyper-rectangle $\mathcal{R}(\overline{\boldsymbol{p}}, \boldsymbol{m})$ is defined by

$$
\mathcal{R}(\overline{\boldsymbol{p}}, \boldsymbol{m})=\{\boldsymbol{p}: \overline{\boldsymbol{p}}-\boldsymbol{m} \leq \boldsymbol{p} \leq \overline{\boldsymbol{p}}+\boldsymbol{m}]\} .
$$

For the sake of clarity, the presentation that follows concentrates on the case where the nominal design point belongs to $\mathcal{E}$, i.e., when the controller satisfies the requirements for the nominal plant. One of the tasks of interest is to assign a measure of robustness to a controller based on measuring how much the reference set can be deformed before intersecting the failure domain. This requires specifying what we mean by a deformation. The Homothetic Deformation of $\Omega$ with respect to the nominal parameter point $\overline{\boldsymbol{p}}$ by a factor of $\alpha \geq 0$, is the set $\mathcal{H}(\Omega, \alpha) \triangleq\{\overline{\boldsymbol{p}}+\alpha(\boldsymbol{p}-\overline{\boldsymbol{p}}): \boldsymbol{p} \in \Omega\}$. The factor of this deformation, $\alpha$, is called the Similitude Ratio. While expansions are accomplished 
when $\alpha>1$, contractions result when $0 \leq \alpha<1$. Hereafter, deformations must be interpreted as homothetic expansions or contractions. For purposes of this paper, two uncertainty sets will be called Proportional if there exist a homothetic deformation that relates them, e.g., $\mathcal{R}(\overline{\boldsymbol{p}}, \boldsymbol{m})$ and $\mathcal{R}(\overline{\boldsymbol{p}}, \alpha \boldsymbol{m})$ are proportional sets since $\mathcal{H}(\mathcal{R}(\overline{\boldsymbol{p}}, \boldsymbol{m}), \alpha)=\mathcal{R}(\overline{\boldsymbol{p}}, \alpha \boldsymbol{m})$.

Intuitively, one imagines that a set proportional to the reference set is being deformed with respect to the nominal parameter point until its boundary touches the boundary of the failure domain, i.e., until at least one member of the deformed set is at the verge of violating one or more closed-loop requirements. A point where the deforming set touches the failure domain is a Critical Parameter Value (CPV). The CPV, which will be denoted as $\tilde{\boldsymbol{p}}$, might not be unique. The deformed set is called the Maximal Set (MS) and will be denoted as $\mathcal{M}$. The Critical Similitude Ratio (CSR), denoted as $\tilde{\alpha}$, is the similitude ratio of that deformation. The CSR is a non-dimensional metric that quantifies the size of the MS, while the Parametric Safety Margin (PSM) ${ }^{14}$ is its dimensional equivalent. Formulations that enable the deformation of hyper-spherical and hyper-rectangular sets are available. ${ }^{14}$ Those corresponding to the latter are presented next.

Recall that the infinity norm is defined as $\|\boldsymbol{x}\|^{\infty} \triangleq \sup _{i}\left\{\left|\boldsymbol{x}_{i}\right|\right\}$. Let us define the scaled infinity norm as $\|\boldsymbol{x}\|_{\boldsymbol{m}}^{\infty} \triangleq \sup _{i}\left\{\left|\boldsymbol{x}_{i}\right| / \boldsymbol{m}_{i}\right\}$. The deformation of the reference set $\mathcal{R}(\overline{\boldsymbol{p}}, \boldsymbol{m})$ when $\overline{\boldsymbol{d}} \in \mathcal{E}$ leads to the following expression for the CPV of the $j$ th requirement

$$
\left\langle\tilde{\boldsymbol{p}}^{j}, \tilde{\alpha}^{j}\right\rangle=\underset{\boldsymbol{p}, \alpha}{\operatorname{argmin}}\left\{\alpha: \boldsymbol{g}_{j}(\boldsymbol{p}, \overline{\boldsymbol{d}}) \geq 0, \overline{\boldsymbol{p}}-\alpha \boldsymbol{m} \leq \boldsymbol{p} \leq \overline{\boldsymbol{p}}+\alpha \boldsymbol{m}\right\},
$$

The overall CPV and CSR are given by

$$
\begin{aligned}
& \tilde{\boldsymbol{p}}=\tilde{\boldsymbol{p}}^{k}, \\
& \tilde{\alpha}=\tilde{\alpha}^{k},
\end{aligned}
$$

where

$$
k=\underset{1 \leq j \leq \operatorname{dim}(\boldsymbol{g})}{\operatorname{argmin}}\left\{\left\|\tilde{\boldsymbol{p}}^{j}-\overline{\boldsymbol{p}}\right\|_{\boldsymbol{m}}^{\infty}\right\} .
$$

On the other hand, if $\overline{\boldsymbol{d}} \notin \mathcal{E}$, we have

$$
\tilde{\boldsymbol{p}}^{j}=\underset{\boldsymbol{p}}{\operatorname{argmin}}\left\{\|\boldsymbol{p}-\overline{\boldsymbol{p}}\|_{\boldsymbol{m}}^{\infty}: \boldsymbol{g}_{j}(\boldsymbol{p}, \overline{\boldsymbol{d}}) \leq 0\right\},
$$

and

$$
\tilde{\boldsymbol{p}}=\underset{\boldsymbol{p}}{\operatorname{argmin}}\left\{\|\boldsymbol{p}-\overline{\boldsymbol{p}}\|_{\boldsymbol{m}}^{\infty}: \boldsymbol{g}_{j}(\boldsymbol{p}, \overline{\boldsymbol{d}}) \leq 0, j=1, \ldots, \operatorname{dim}(\boldsymbol{g})\right\},
$$

One might argue that the solution to the last two equations is unnecessary since $\overline{\boldsymbol{d}}$ does not even satisfy the design requirements for the nominal plant (i.e., plant evaluated at the nominal parameter point). However, one situation in which a need for this extension might arise is if an automated, optimization driven design procedure varies the design parameter so much that constraint boundaries move enough to make $\overline{\boldsymbol{p}}$ a constraint violation point (as in Section IV). Once the CPV has been found, the MS is uniquely determined. In this case, the corresponding MS is given by

$$
\mathcal{M}_{\boldsymbol{p}}=\mathcal{R}(\overline{\boldsymbol{p}}, \tilde{\alpha} \boldsymbol{m})
$$

When the uncertainty model is probabilistic, a natural quantifier of robustness is the probability of violating the closed-loop requirements. This probability, called the Failure Probability, will be denoted as $P[\mathcal{F}]$. The formulation above, which enables the deformation of sets in $\boldsymbol{p}$-space, can be extended to the standard normal space, called the $\boldsymbol{u}$-space, via the probability preserving 
transformation $\boldsymbol{u}=U(\boldsymbol{p})$. In this setting, the deformation of the reference set $\mathcal{R}(\overline{\boldsymbol{u}}, \boldsymbol{m})$ when $\overline{\boldsymbol{d}} \in \mathcal{E}$ leads to the following expression for the $\mathrm{CPV}$ of the $j$ th requirement

$$
\left\langle\tilde{\boldsymbol{u}}^{j}, \tilde{\alpha}^{j}\right\rangle=\underset{\boldsymbol{u}, \alpha}{\operatorname{argmin}}\left\{\alpha: \boldsymbol{g}_{j}\left(U^{-1}(\boldsymbol{u}), \overline{\boldsymbol{d}}\right) \geq 0, \overline{\boldsymbol{u}}-\alpha \boldsymbol{m} \leq \boldsymbol{u} \leq \overline{\boldsymbol{u}}+\alpha \boldsymbol{m}\right\} .
$$

The overall CPV and CSR are given by

$$
\begin{aligned}
& \tilde{\boldsymbol{u}}=\tilde{\boldsymbol{u}}^{k}, \\
& \tilde{\alpha}=\tilde{\alpha}^{k},
\end{aligned}
$$

where

$$
k=\underset{1 \leq j \leq \operatorname{dim}(\boldsymbol{g})}{\operatorname{argmin}}\left\{\left\|\tilde{\boldsymbol{u}}^{j}-\overline{\boldsymbol{u}}\right\|_{\boldsymbol{m}}^{\infty}\right\} .
$$

The case when $\overline{\boldsymbol{d}} \notin \mathcal{E}$ can be easily inferred from Equations (12-13). In this context, the corresponding MS is given by

$$
\mathcal{M}_{\boldsymbol{u}}=\mathcal{R}(\overline{\boldsymbol{u}}, \tilde{\alpha} \boldsymbol{m}) .
$$

Analogous to the PSM in $\boldsymbol{p}$-space, the Reliability Index (RI $)^{14}$ is a dimensional metric proportional to the size of this set. Throughout the developments of this section the design point $\overline{\boldsymbol{d}}$ has been kept fixed while sets in the original parameter space $\boldsymbol{p}$, or its transformed version $\boldsymbol{u}$, have been deformed. In what follows we extend these ideas to settings where the deformations take place in the design space or in the union of both the parameter and the design spaces.

\section{Analysis of the Design Space}

In this section, we develop strategies for finding lower bounds of the feasible design space $\mathcal{E}$, the robust design space $\mathcal{Q}$, and the $(1-\epsilon)$-probable design space $\mathcal{D}$, corresponding to a fixed control structure. By using these bounds as constraints, the search for optimal controllers within these sets can be efficiently performed, e.g., searching for a controller that minimizes the variability in the system response given that $P[\boldsymbol{g}(\boldsymbol{p}, \boldsymbol{d})>\mathbf{0}]<\epsilon$. We assume that a baseline controller with parameters $\overline{\boldsymbol{d}}$ is available, and the design specifications in $\boldsymbol{g}$ are given. The presentation that follows only considers the deformation of hyper-rectangular sets.

\section{III.A. Bounding the Feasible Design Space}

A lower bound for the feasible design space $\mathcal{E}$ is attained by deforming the reference set $\mathcal{R}(\overline{\boldsymbol{d}}, \boldsymbol{n})$ about $\overline{\boldsymbol{d}}$ until the deformed set touches the failure region $\mathcal{F}_{\boldsymbol{d}}(\overline{\boldsymbol{p}})$. In the process, the uncertain parameter is kept fixed at its nominal value. A natural parallelism between the concepts and formulations used for deforming sets in the parameter space and those to be used for deforming sets in the design space is apparent. For instance, the roles of the nominal parameter point $\bar{p}$, the nominal design point $\overline{\boldsymbol{d}}$, and the CPV $\tilde{\boldsymbol{p}}$ will now be assumed by the nominal design point $\overline{\boldsymbol{d}}$, the nominal parameter point $\overline{\boldsymbol{p}}$, and the Critical Design Value (CDV), $\tilde{\boldsymbol{d}}$, respectively.

The critical design value and the CSR for the $j$ th requirement are given by

$$
\left\langle\tilde{\boldsymbol{d}}^{j}, \tilde{\alpha}^{j}\right\rangle=\underset{\boldsymbol{d}, \alpha}{\operatorname{argmin}}\left\{\alpha: \boldsymbol{g}_{j}(\overline{\boldsymbol{p}}, \boldsymbol{d}) \geq 0, \overline{\boldsymbol{d}}-\alpha \boldsymbol{n} \leq \boldsymbol{d} \leq \overline{\boldsymbol{d}}+\alpha \boldsymbol{n}\right\} .
$$

The overall CDV and CSR are given by

$$
\begin{aligned}
& \tilde{\boldsymbol{d}}=\tilde{\boldsymbol{d}}^{k}, \\
& \tilde{\alpha}=\tilde{\alpha}^{k},
\end{aligned}
$$


where

$$
k=\underset{1 \leq j \leq \operatorname{dim}(\boldsymbol{g})}{\operatorname{argmin}}\left\{\left\|\tilde{\boldsymbol{d}}^{j}-\overline{\boldsymbol{d}}\right\|_{\boldsymbol{n}}^{\infty}\right\} .
$$

Hence, the overall CDV is found by solving for the CDV of each individual requirement, and selecting the closest to the nominal design point according to the scaled infinity norm. The MS resulting from this deformation is given by

$$
\mathcal{M}_{\boldsymbol{d}}=R(\overline{\boldsymbol{d}}, \tilde{\alpha} \boldsymbol{n}) .
$$

The MS is the largest hyper-rectangle proportional to $\mathcal{R}(\overline{\boldsymbol{d}}, \boldsymbol{n})$ which fits within the feasible design space. Therefore, all the gains within $\mathcal{M}_{\boldsymbol{d}}$ satisfy the closed-loop specifications for the nominal plant.

\section{III.B. Bounding the Robust Design Space}

A formulation that enables bounding the robust design space $\mathcal{Q}$ is presented next. In what follows, we assume that the uncertainty set $\Delta=\mathcal{R}(\overline{\boldsymbol{p}}, \boldsymbol{m})$ is given, and that the nominal design point $\overline{\boldsymbol{d}}$ belongs to $\mathcal{Q}$. The latter assumption holds when $\Delta$ is a subset of the MS in Equation (14). In contrast to the deformations presented thus far, the one required for bounding $\mathcal{Q}$ will take place in both the parameter and the design spaces. Analogous to the CPV and the CDV, the Critical Pair $\langle\tilde{\boldsymbol{p}}, \tilde{\boldsymbol{d}}\rangle$, made of a parameter point(s) and a design point(s), results from deforming the reference set $\Omega=\Delta \cup \mathcal{R}(\overline{\boldsymbol{d}}, \boldsymbol{n})$, i.e., $\{\langle\boldsymbol{p}, \boldsymbol{d}\rangle: \boldsymbol{p} \in \Delta, \boldsymbol{d} \in \mathcal{R}(\overline{\boldsymbol{d}}, \boldsymbol{n})\}$, in the $\boldsymbol{d}$ directions until the deformed set touches the failure region $\mathcal{F}_{\boldsymbol{p}, \boldsymbol{d}}$. Note that while $\boldsymbol{m}$ depends on the prescribed uncertainty set $\Delta$, $\boldsymbol{n}$ is arbitrary.

The critical pair and the CSR for the $j$ th requirement are given by

$$
\left\langle\tilde{\boldsymbol{p}}^{j}, \tilde{\boldsymbol{d}}^{j}, \tilde{\alpha}^{j}\right\rangle=\underset{\boldsymbol{p}, \boldsymbol{d}, \alpha}{\operatorname{argmin}}\left\{\alpha: \boldsymbol{g}_{j}(\boldsymbol{p}, \boldsymbol{d}) \geq 0, \overline{\boldsymbol{p}}-\boldsymbol{m}<\boldsymbol{p}<\overline{\boldsymbol{p}}+\boldsymbol{m}, \overline{\boldsymbol{d}}-\alpha \boldsymbol{n} \leq \boldsymbol{d} \leq \overline{\boldsymbol{d}}+\alpha \boldsymbol{n}\right\} .
$$

The overall critical pair and the overall CSR are given by

$$
\begin{aligned}
\langle\tilde{\boldsymbol{p}}, \tilde{\boldsymbol{d}}\rangle & =\left\langle\tilde{\boldsymbol{p}}^{k}, \tilde{\boldsymbol{d}}^{k}\right\rangle, \\
\tilde{\alpha} & =\tilde{\alpha}^{k}
\end{aligned}
$$

where

$$
k=\underset{1 \leq j \leq \operatorname{dim}(\boldsymbol{g})}{\operatorname{argmin}}\left\{\tilde{\alpha}^{j}\right\} .
$$

Hence, the overall critical pair is found by solving for the critical pair of each individual requirement, and selecting the one attaining the smallest CSR. The MS resulting from this deformation is given by

$$
\mathcal{M}_{\boldsymbol{p}, \boldsymbol{d}}=\Delta \cup \mathcal{R}(\overline{\boldsymbol{d}}, \tilde{\alpha} \boldsymbol{n}) .
$$

Note that the projection of this MS into the design space is a subset of the robust design space. Therefore, all the gain vectors within the set $\mathcal{R}(\overline{\boldsymbol{d}}, \tilde{\alpha} \boldsymbol{n})$ satisfy the design specifications for all the parameter realizations in $\Delta$.

A sketch illustrating relevant quantities is shown in Figure 2. Note that the reference set $\Omega$ is a set in $\boldsymbol{p} \cup \boldsymbol{d}$-space centered at $\langle\overline{\boldsymbol{p}}, \overline{\boldsymbol{d}}\rangle$ whose projection into $\boldsymbol{p}$-space is $\Delta=\mathcal{R}(\overline{\boldsymbol{p}}, \boldsymbol{m})$. The deformation of the reference set in the $\boldsymbol{d}$ direction leads to the critical pair $\langle\tilde{\boldsymbol{p}}, \tilde{\boldsymbol{d}}\rangle$ and to the MS $\mathcal{M}_{\boldsymbol{p}, \boldsymbol{d}}$. Since the projection of the MS into $\boldsymbol{p}$-space is $\Delta$, all the designs in $\mathcal{R}(\overline{\boldsymbol{d}}, \tilde{\alpha} \boldsymbol{n})$ satisfy the requirement $\boldsymbol{g}(\boldsymbol{p}, \boldsymbol{d}) \leq \mathbf{0}, \forall \boldsymbol{p} \in \Delta$. Further, notice that the projection of the MS into $\boldsymbol{d}$-space is a subset of $\mathcal{Q}(\Delta)$. 


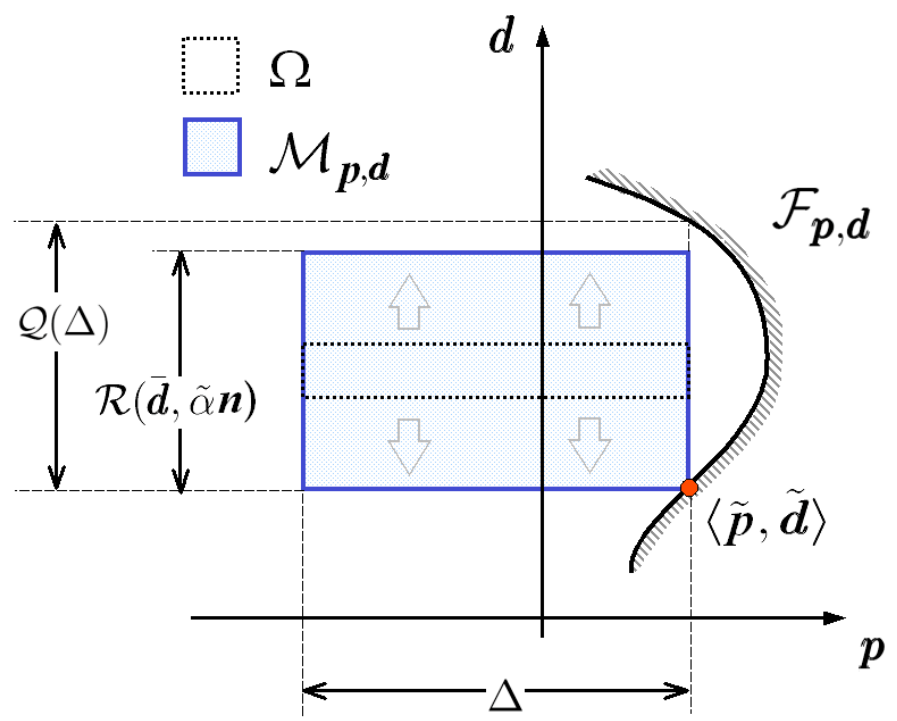

Figure 2. Relevant metrics in the bounding of the robust design space.

\section{III.C. Bounding the $(1-\epsilon)$-Probable Design Space}

A formulation that enables bounding the $(1-\epsilon)$-probable design space $\mathcal{D}\left(f_{\boldsymbol{p}}, \epsilon\right)$ for a given value of $\epsilon$ is presented next. In what follows we assume that a probabilistic uncertainty model is available. Let us call the Exclusion Set, $\mathcal{X}$, a set in the parameter space that satisfies $P[\mathcal{X}]=1-\epsilon$. Clearly, $\mathcal{X}$ is not unique. The following expressions, derived in detail in the companion paper [14], enable the calculation of several exclusion sets in $\boldsymbol{p}$ - and $\boldsymbol{u}$-spaces. The first one is

$$
\mathcal{X}_{\boldsymbol{p}}=\mathcal{R}\left(\overline{\boldsymbol{p}}, \delta \frac{\boldsymbol{m}}{\|\boldsymbol{m}\|}\right)
$$

where the value of $\delta$ is given by

$$
\prod_{i=1}^{\operatorname{dim}(\boldsymbol{p})} \boldsymbol{F}_{\boldsymbol{p}_{i}}\left(\overline{\boldsymbol{p}}_{i}+\frac{\delta \boldsymbol{m}_{i}}{\|\boldsymbol{m}\|}\right)-\boldsymbol{F}_{\boldsymbol{p}_{i}}\left(\overline{\boldsymbol{p}}_{i}-\frac{\delta \boldsymbol{m}_{i}}{\|\boldsymbol{m}\|}\right)=1-\epsilon,
$$

and $\boldsymbol{F}_{\boldsymbol{p}}$ is the cumulative distribution function associated with $\boldsymbol{f}_{\boldsymbol{p}}$. In this expression, the values of $\overline{\boldsymbol{p}}$ and $\boldsymbol{m}$ are up to the analyst. Alternatively, we can also use

$$
\mathcal{X}_{\boldsymbol{u}}=\mathcal{S}(\mathbf{0}, \delta)
$$

where the value of $\delta$ is given by

$$
\Lambda_{l}(\delta)=1-\epsilon
$$

where

$$
\Lambda_{l}(\delta)= \begin{cases}\operatorname{erf}\left(\frac{\delta}{\sqrt{2}}\right)-\sqrt{\frac{2}{\pi}}\left(\frac{\delta^{l-2}}{(l-2) ! !}+\frac{\delta^{l-4}}{(l-4) ! !}+\cdots+\frac{\delta}{1 ! !}\right) e^{-\delta^{2} / 2} & \text { if } \delta \geq 0, l \text { odd } \\ 1-\left(\frac{\delta^{l-2}}{(l-2) ! !}+\frac{\delta^{l-4}}{(l-4) ! !}+\cdots+\frac{\delta^{2}}{2 ! !}+1\right) e^{-\delta^{2} / 2} & \text { if } \delta \geq 0, l \text { even } \\ 0 & \text { otherwise }\end{cases}
$$


$l=\operatorname{dim}(\boldsymbol{p})$, and $n ! !$ is the double factorial ${ }^{\mathrm{c}}$. In addition, we may also consider

$$
\mathcal{X}_{u}=\mathcal{R}\left(\bar{u}, \delta \frac{\boldsymbol{m}}{\|\boldsymbol{m}\|}\right)
$$

where the value of $\delta$ is given by

$$
\prod_{i=1}^{\operatorname{dim}(\boldsymbol{p})} \Phi\left(\overline{\boldsymbol{u}}_{i}+\frac{\delta \boldsymbol{m}_{i}}{\|\boldsymbol{m}\|}\right)-\Phi\left(\overline{\boldsymbol{u}}_{i}-\frac{\delta \boldsymbol{m}_{i}}{\|\boldsymbol{m}\|}\right)=1-\epsilon
$$

and $\Phi$ is the cumulative distribution function of the univariate standard normal random variable. In the latter expression, $\overline{\boldsymbol{u}}$ and $\boldsymbol{m}$ are up to the analyst.

While Equation (25) only applies to the case of independent random variables the other two require the $U$ transformation. Note that the hyper-spherical set centered at the origin of the standard normal space contains the largest probability per unit of volume. This implies that $\mathcal{X}_{\boldsymbol{u}}$ in Equation (26) is the set in $\boldsymbol{u}$-space of smallest volume whose probability is $1-\epsilon$.

Note that all the design points that are robust to $\Delta=\mathcal{X}$, satisfy the chance constraint in Equation (7). The bounding of $\mathcal{D}$ will be performed by searching for a MS in $\boldsymbol{p} \cup \boldsymbol{d}$-space whose projection into the parameter space is the exclusion set. As with the bounding of the robust design space, we assume that the nominal design point $\overline{\boldsymbol{d}}$ belongs to $\mathcal{D}$. The membership of $\overline{\boldsymbol{d}}$ in $\mathcal{D}$ is guaranteed when the probability of the MS in Equation (14) is larger than $1-\epsilon$. In the presentation that follows we will only consider exclusion sets in $\boldsymbol{u}$-space. As before, the critical pair $\langle\tilde{\boldsymbol{u}}, \tilde{\boldsymbol{d}}\rangle$ results from deforming the reference set $\Omega=\mathcal{X}_{\boldsymbol{u}} \cup \mathcal{R}(\overline{\boldsymbol{d}}, \boldsymbol{n})$ in the $\boldsymbol{d}$ directions until the deformed set touches the failure region $U\left(\mathcal{F}_{\boldsymbol{p}, \boldsymbol{d}}\right)$. In this context, the critical pair and the CSR for the $j$ th requirement are given by

$$
\left\langle\tilde{\boldsymbol{u}}^{j}, \tilde{\boldsymbol{d}}^{j}, \tilde{\alpha}^{j}\right\rangle=\underset{\boldsymbol{u}, \boldsymbol{d}, \alpha}{\operatorname{argmin}}\left\{\alpha: \boldsymbol{g}_{j}\left(U^{-1}(\boldsymbol{u}), \boldsymbol{d}\right) \geq 0, \boldsymbol{u} \in \mathcal{X}_{\boldsymbol{u}}, \overline{\boldsymbol{d}}-\alpha \boldsymbol{n} \leq \boldsymbol{d} \leq \overline{\boldsymbol{d}}+\alpha \boldsymbol{n}\right\} .
$$

The second constraint is equal to $\|\boldsymbol{u}\|<\delta$ when the exclusion set is hyper-spherical and to $\| \boldsymbol{u}-$ $\overline{\boldsymbol{u}}\left\|_{\boldsymbol{m}}^{\infty}\right\| \boldsymbol{m} \|<\delta$ when it is hyper-rectangular.

The overall critical pair and CSR are also given by Equation (23). The MS resulting from this deformation is given by

$$
\mathcal{M}_{\boldsymbol{u}, \boldsymbol{d}}=\mathcal{X}_{\boldsymbol{u}} \cup \mathcal{R}(\overline{\boldsymbol{d}}, \tilde{\alpha} \boldsymbol{n}) .
$$

The projection of the MS into the design space is a subset of the $(1-\epsilon)$-probable design space. Therefore, all the gain vectors within the set $\mathcal{R}(\overline{\boldsymbol{d}}, \tilde{\alpha} \boldsymbol{n})$ satisfy the closed-loop specifications with probability of at least $1-\epsilon$.

A sketch illustrating relevant quantities is shown in Figure 3. The bottom plot shows that the probability of the exclusion set is $1-\epsilon$ by construction. While this plot shows the probability density function in $\boldsymbol{u}$-space, the one in the top shows the $\boldsymbol{u} \cup \boldsymbol{d}$-space. Note that the projection of the reference set $\Omega$, which is centered at $\langle\mathbf{0}, \overline{\boldsymbol{d}}\rangle$, into $\boldsymbol{u}$-space is the exclusion set $\mathcal{X}_{\boldsymbol{u}}$. The deformation of the reference set in the $\boldsymbol{d}$ direction leads to the critical pair $\langle\tilde{\boldsymbol{u}}, \tilde{\boldsymbol{d}}\rangle$ and to the MS $\mathcal{M}_{\boldsymbol{u}, \boldsymbol{d}}$. Since the projection of the MS into $\boldsymbol{u}$-space is the exclusion set, all the designs in $\mathcal{R}(\overline{\boldsymbol{d}}, \tilde{\alpha} \boldsymbol{n})$ satisfy the requirement $P[\boldsymbol{g}(\boldsymbol{p}, \boldsymbol{d})>\mathbf{0}] \leq \epsilon$. Further notice that the projection of the MS into $\boldsymbol{d}$-space is a subset of $\mathcal{D}\left(f_{\boldsymbol{p}}, \epsilon\right)$.

\footnotetext{
${ }^{\mathrm{c}}$ Recall that the double factorial is defined as

$$
n ! != \begin{cases}n \cdot(n-2) \cdots 5 \cdot 3 \cdot 1 & n>0 \text { and odd } \\ n \cdot(n-2) \cdots 6 \cdot 4 \cdot 2 & n>0 \text { and even } \\ 1 & n=-1,0\end{cases}
$$
}




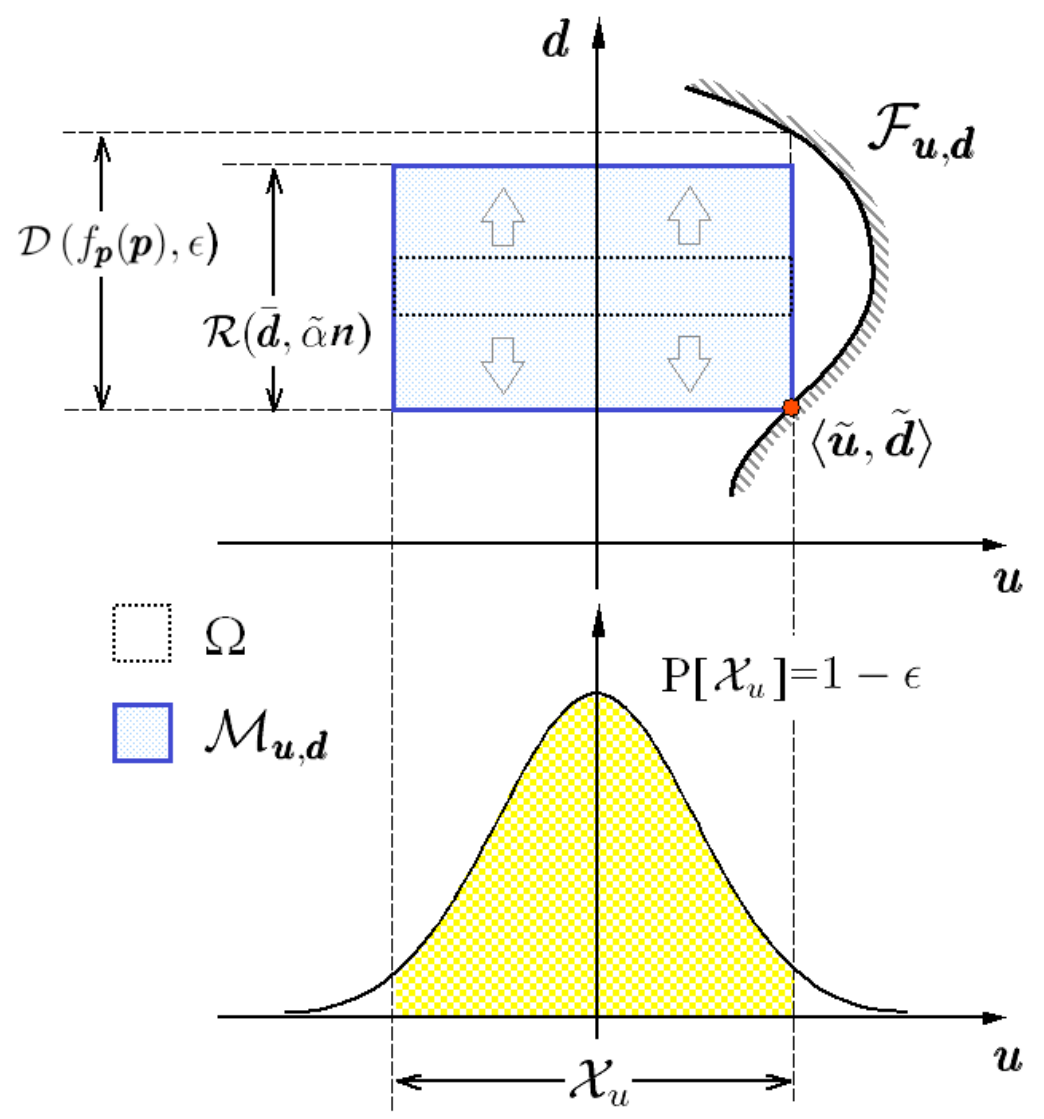

Figure 3. Relevant metrics in the bounding of the $(1-\epsilon)$-probable design space

\section{Control Tuning}

In this section we seek to improve the robustness characteristics of a baseline controller by tuning its gains. In principle, the targeted controllers will realize the largest $\mathcal{M}_{\boldsymbol{p}}$, or $\mathcal{M}_{\boldsymbol{u}}$ the control structure allows. Note that these maximal sets are those from Equations (14) and (18), not those from Section III. The three formulations to be presented will evaluate the robustness characteristics of any design point considered during the search for the optimum (i) by sizing the exact MS, (ii) by using a multi-point approximation to the MS, and (iii) by using a multi-point evaluation of the constraint function. Only the first of these three strategies always leads to the intended designs. The other two may not due to the approximate nature of the formulation. However, their relaxed computational demands make them attractive in spite of their potential drawbacks. Because the framework in Reference [14] allows for a rigorous analysis of any given design point, the inaccuracies resulting from such drawbacks can be detected a posteriori.

\section{IV.A. Maximization of the Critical Similitude Ratio}

This problem of interest is given by

$$
\boldsymbol{d}^{*}=\underset{\boldsymbol{d}}{\operatorname{argmax}}\{\gamma \tilde{\alpha}(\boldsymbol{d})\}
$$

where $\tilde{\alpha}$ is the CSR in Equation (10) or Equation (17), and $\gamma=1$ if $\boldsymbol{d} \in \mathcal{E}$, otherwise $\gamma=-1$. Figure 1 illustrates the optimal design $\boldsymbol{d}^{*}$ on a one-dimensional setting. Recall that determining 
the $\tilde{\alpha}$ corresponding to any given design point entails solving an optimization problem. Therefore, in contrast to all the problem formulations posed thus far, this one has an optimization in the outer loop and another one in the inner loop. While the outer loop searches for the best set of gains $\boldsymbol{d}^{*}$, the inner one searches for the overall CPV, i.e., $\tilde{\boldsymbol{p}}$ or $\tilde{\boldsymbol{u}}$, corresponding to the design point under evaluation.

The nested optimization in Equation (30), which typically results from worst-case-based design policies, usually imposes stringent computational demands. Multi-point approaches can be used to reduce the computational burden associated with solving Equation (30). The multi-point approaches in the next two sections may result in designs which are suboptimal or infeasible. However, these design points can be used as initial guesses when solving Equation (30). Numerical experiments showed that this practice usually results in more rapid convergence and less computational burden.

\section{IV.B. Expansion of an Approximate MS}

Even though the strategy considered herein is applicable to sets in both $\boldsymbol{p}$ - and $\boldsymbol{u}$-spaces, only the case in $\boldsymbol{u}$-space will be presented. An approximation to the solution of Equation (30) is given by

$$
\left\langle\boldsymbol{d}^{*}, \delta^{*}\right\rangle=\underset{\boldsymbol{d}, \delta}{\operatorname{argmax}}\left\{\delta: \max _{j, i}\left\{\boldsymbol{g}_{j}\left(U^{-1}\left(\overline{\boldsymbol{u}}+\delta \boldsymbol{u}^{i}\right), \boldsymbol{d}\right)\right\}<0, \delta \geq 0\right\},
$$

where $\boldsymbol{u}^{i}$ for $i=1, \cdots n$ are parameter-points on the surface of either $\mathcal{S}(\mathbf{0}, 1)$ or $\mathcal{R}(\mathbf{0}, \boldsymbol{m})$. Ideally, such points, which only have to be computed once, should be uniformly distributed over the chosen surface. Note that this formulation replaces the inner optimization loop in Equation (30) by a multi-point constraint over parameter points lying on the surface of the approximate MS.

Since the satisfaction of the multi-point constraints does not guarantee the enforcement of the true requirement $\mathcal{M} \subset \mathcal{F}_{\boldsymbol{u}, \boldsymbol{d}}^{c}$, this formulation may lead to suboptimal designs. Besides, the larger the value of $\delta$, the smaller the density of points over the surface of the approximated MS, and the greater the chance to converge to an overly large approximation of the true MS.

Procedures to generate the $\boldsymbol{u}^{i}$ points are presented next. For the hyper-spherical case, the desired points result from generating $n$ samples of an uncorrelated standard normal vector, and then scale them to have unit length. The resulting points not only lie on the surface of a unit sphere centered at the origin but will also be uniformly distributed over that surface. Now consider the hyper-rectangular case. Let $\boldsymbol{q}^{i}$ be a sample of points distributed uniformly over the surface of the unit sphere as the $\boldsymbol{u}^{i}$ chosen for the hyper-spherical case. Each $\boldsymbol{q}^{i}$ will be projected radially onto the surface $\mathcal{R}(\mathbf{0}, \boldsymbol{m})$. Since this surface is characterized by $\|\boldsymbol{u}\|_{\boldsymbol{m}}^{\infty}=1$, the desired point is

$$
\boldsymbol{u}^{i}=\frac{\boldsymbol{q}^{i}}{\left\|\boldsymbol{q}^{i}\right\|_{\boldsymbol{m}}^{\infty}}
$$

The concentration of points resulting from this scheme increases with the closeness of the surface to the origin. Therefore, there will be a lower density of samples in the vicinity of the corners of the hyper-rectangle. An alternative approach, whose sample points are more evenly distributed over the surface of the hyper-rectangle, is as follows. The points $\boldsymbol{u}^{i}$ can also be obtained after mapping the $\boldsymbol{q}^{i}$ s through the inverse of the Q-transformation[15], which is given by

$$
Q^{-1}(\boldsymbol{q})=\frac{\|\boldsymbol{q}\|}{\max \{|\boldsymbol{q}|\}} \operatorname{diag}\{\boldsymbol{m}\} \boldsymbol{q} .
$$

This scheme leads to a parameter point set that has approximately the same number of points in each face of $\mathcal{R}(\mathbf{0}, \boldsymbol{m})$. In contrast to the previous distribution, the concentration of points in a given face of the hyper-rectangle will be higher closer to the edges. 


\section{IV.C. Multi-point Constraint Minimization}

In this formulation we search for a control design that minimizes the largest value of the constraint function at a set of fixed parameter points. Let the points $\boldsymbol{u}^{i}$ for $i=1, \ldots, n$ be on the surface of an arbitrary set in the parameter space. Points on the surface of hyper-spherical and hyperrectangular sets can be obtained by scaling the points resulting from applying the algorithms of the previous section. We would like for all the members of such a set to be well into the non-failure region, $U\left(\mathcal{F}_{\boldsymbol{p}}^{c}(\boldsymbol{d})\right)$. In this context, the formulation of interest is given by

$$
\boldsymbol{d}^{*}=\underset{\boldsymbol{d}}{\operatorname{argmin}}\left\{\max _{j, i}\left\{\boldsymbol{g}_{j}\left(U^{-1}\left(\boldsymbol{u}^{i}\right), \boldsymbol{d}\right)\right\}\right\}
$$

This equation has the minimax structure also used in Reference [8]. Note that points in the feasible design space can be identified by using $\overline{\boldsymbol{u}}$ as one of the parameter points and attaining $\boldsymbol{g}\left(U^{-1}(\overline{\boldsymbol{u}}), \boldsymbol{d}^{*}\right) \leq \mathbf{0}$. This formulation uses the worst-case value of the constraint function at the sampled points to approximate the separation between the set whose surface is being sampled and the failure domain; i.e., the more negative the value of $\boldsymbol{g}$ at the sampled points the further the failure domain is from the set. Obviously, this approximation is not good in general. For this and the previous formulation, it is easy to foresee situations leading to unacceptable designs, e.g., having a failure domain that extends to the interior of the sampled set but for which $\boldsymbol{g}\left(U^{-1}\left(\boldsymbol{u}^{i}\right), \boldsymbol{d}^{*}\right) \leq \mathbf{0}$ for $i=1, \ldots, n$. When cases like this arise, one should add the CPV corresponding to a faulty design to the set of parameter points to be used in subsequent searches.

\section{IV.D. Discussion}

Control design formulations aiming at the minimization of the failure probability are available $[12,13,10]$. Numerical difficulties arise when these formulations use sampling-based approximations to $P[\mathcal{F}]$. This occurs because the approximation is a piecewise constant, discontinuous, and nonsmooth function of the design variable, properties which cause problems when derivatives are required. Note that the calculation of $P[\mathcal{F}]$ does not require the definition of a nominal parameter point, and designs attaining small failure probabilities may have parts of their failure domain well inside $\Delta$. Designs attaining both a small $P[\mathcal{F}]$ and a large separation between $\overline{\boldsymbol{p}}$ and $\mathcal{F}$ can be pursued by using the CSR of a RI in Equation (30). This dual notion of robustness can also be attained by searching for a design point $\boldsymbol{d}$ in the set $\mathcal{R}(\overline{\boldsymbol{d}}, \tilde{\alpha} \boldsymbol{n})$ that minimizes $P[\mathcal{F}]$. The set $\mathcal{R}(\overline{\boldsymbol{d}}, \tilde{\alpha} \boldsymbol{n})$ is the bound to $\mathcal{D}$ that results from the deformations of Section III.C. The resulting controller not only minimizes the failure probability but also attains a RI larger or equal to the value of $\delta$ used. The hybrid method of Reference [16] is best suited for the estimation of this probability since the upper bound to $P[\mathcal{F}]$ corresponding to $\mathcal{M}_{\boldsymbol{u}}=\mathcal{X}_{\boldsymbol{u}}$ holds for all design points in $\mathcal{R}(\overline{\boldsymbol{d}}, \tilde{\alpha} \boldsymbol{n})$.

\section{Example: Benchmark Robust Control Problem}

\section{V.A. Problem Statement}

The tuning of a controller designed for the robust control challenge problem ${ }^{17}$ posed in the 1990 American Control Conference is considered next. The control verification of several solutions to this problem is presented in Reference [14]. The benchmark plant, shown in Figure 4, is a twomass/spring system with a non-collocated sensor actuator pair. Several design problems were posed based on this setting. In all of them, stability and performance requirements in the time domain were prescribed for plants with uncertain masses and stiffness whose values lie within a bounded set. As in Reference [18], additional sources of uncertainty are considered herein to fully exercise the 


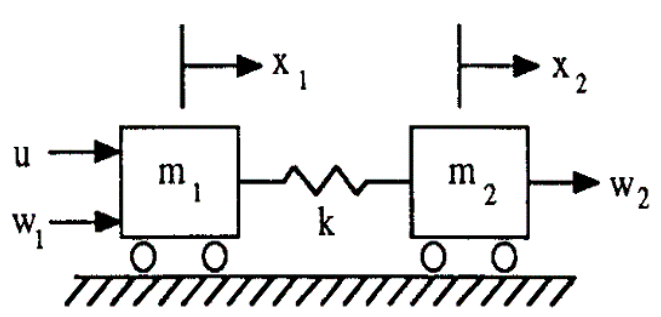

Figure 4. Two-mass spring system.

scope of the methodology. We added a non-linear spring with constant $k_{n}$, a time delay $\tau$ denoting a first order lag between controller command and actuator response, and a loop-gain uncertainty $f$ resulting from multiplicative variation in observation, control gain and/or actuator failure.

The state space plant model is

$$
\begin{aligned}
\dot{x}_{1} & =x_{3} \\
\dot{x}_{2} & =x_{4} \\
\dot{x}_{3} & =\frac{k}{m_{1}}\left(x_{2}-x_{1}\right)+\frac{k_{n}}{m_{1}}\left(x_{2}-x_{1}\right)^{3}+\frac{f u}{m_{1}}, \\
\dot{x}_{4} & =\frac{k}{m_{2}}\left(x_{1}-x_{2}\right)+\frac{k_{n}}{m_{2}}\left(x_{1}-x_{2}\right)^{3}+\frac{w_{2}}{m_{2}}, \\
\tau \dot{u} & =u_{c}-u .
\end{aligned}
$$

While the output $\mathbf{z}$ and the observed variable $\mathbf{y}$ are both equal to $x_{2}$, only the disturbance $w_{2}$ will be active. The uncertain parameter vector is $\boldsymbol{p}=\left[m_{1}, m_{2}, k, k_{n}, \tau, f\right]^{T}$ whose nominal value is $\overline{\boldsymbol{p}}=[1,1,1,0,0,1]^{T}$. Note that the nominal values of the additional parameters lead to the plant used in the original benchmark problem. In order to prevent deformations leading to infeasible plants, the constraints $m_{1}>0, m_{2}>0, k>0, \tau>0$ and $f>0$ are imposed on the optimization problem used to calculate the CPVs.

The specifications imposed on the closed-loop system are:

1. Local closed-loop stability.

2. Settling time: the response to a unit-impulse must fall between \pm 0.1 after $15 \mathrm{~s}$.

3. Control saturation: the control signal corresponding to the impulse response must fall between \pm 1 .

In the context of this paper, the corresponding set of constraints is

$$
\boldsymbol{g}=\left[\max _{1 \leq i \leq n_{p}}\left\{\Re\left(s^{i}\right)\right\}, \quad \max _{t>15}\{|z(t)|\}-0.1, \quad \max _{t>0}\{|u(t)|\}-1\right]^{T},
$$

where $s^{i}$ is a closed-loop pole of the linearized system and $\Re(\cdot)$ is the real part operator. Eleven controllers were designed for the above problem by several authors. The controllers have been design using several different methods, including robust $H_{\infty}$, loop-transfer recovery, imaginaryaxis shifting, constrained optimization, structured covariance, game theory, the internal model principle $^{18,19}$ and $\mu$-synthesis. ${ }^{20}$ A Monte Carlo-based analysis of some of these controllers is available. $^{18}$ 
The state space representation of a controller is given by

$$
\begin{aligned}
\dot{\mathbf{x}}_{c} & =\mathbf{A}_{c} \mathbf{x}_{c}+\mathbf{B}_{c} \mathbf{y}, \\
u_{c} & =\mathbf{C}_{c} \mathbf{x}_{c}+\mathbf{D}_{c} \mathbf{y},
\end{aligned}
$$

where $\mathbf{x}_{c}$ is the controller state, $u_{c}$ is the actuator command, and $\mathbf{A}_{c}, \mathbf{B}_{c}, \mathbf{C}_{c}$, and $\mathbf{D}_{c}$ are the controller matrices. The controllers considered here are the ones labeled as $A, B, C, D, E, F$, and $H$ in Reference [18], and the controllers designed for problems one and two in Reference [19] and Reference [20]. In this paper, the controllers from Reference [19] will be labeled as $W_{1}$ and $W_{2}$, and those from Reference [20] will be labeled as $B_{1}$ and $B_{2}$.
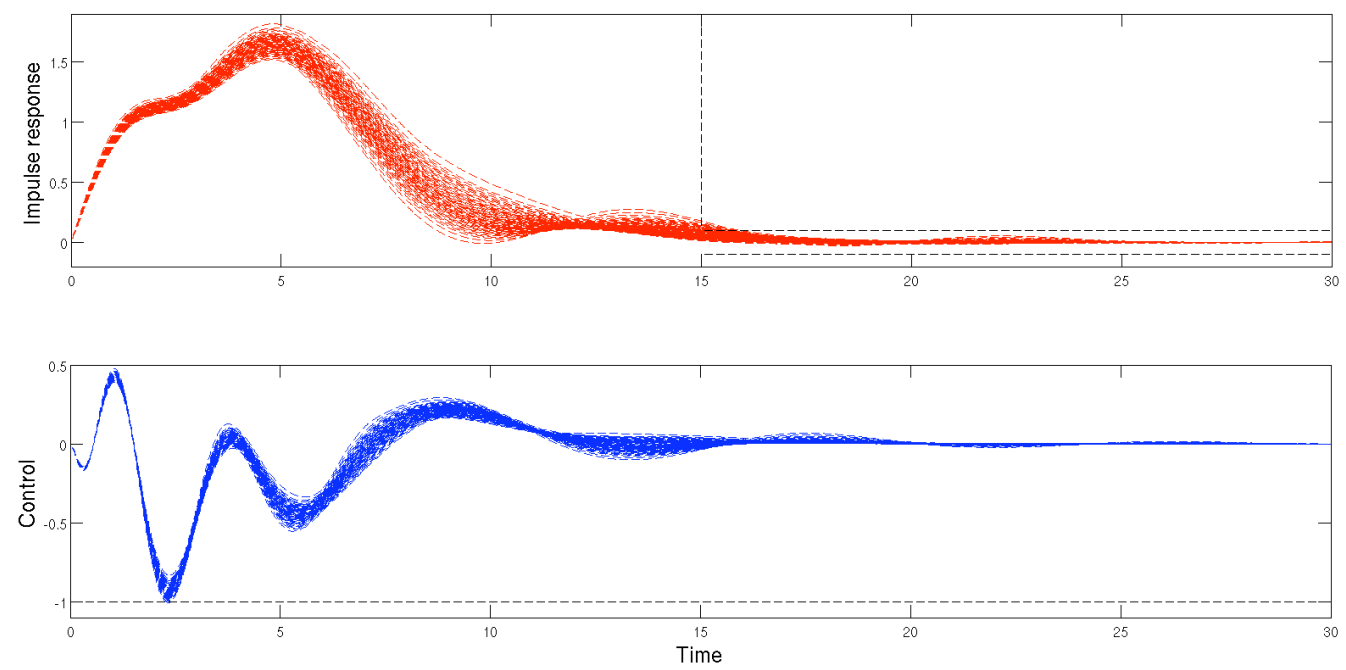

Figure 5. Percentiles $2 \%$ apart of the impulse response and control signal for $B_{2}$.
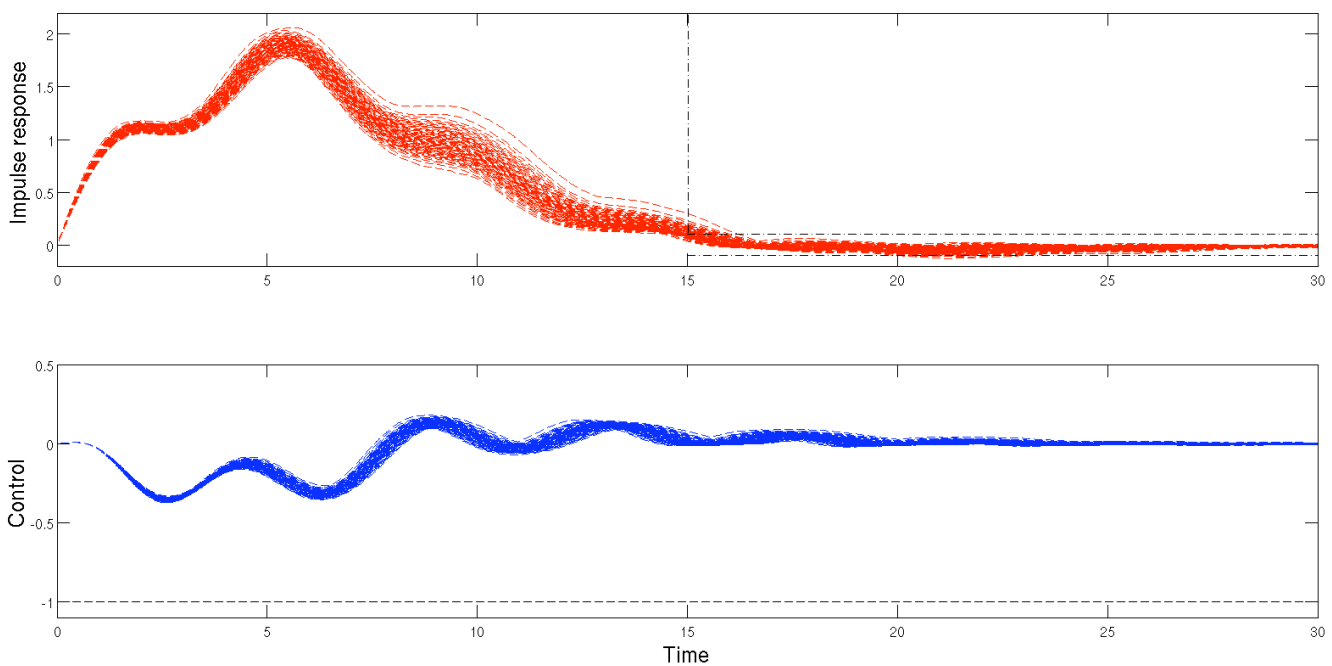

Figure 6. Percentiles $2 \%$ apart of the impulse response and control signal for $W_{2}$. 

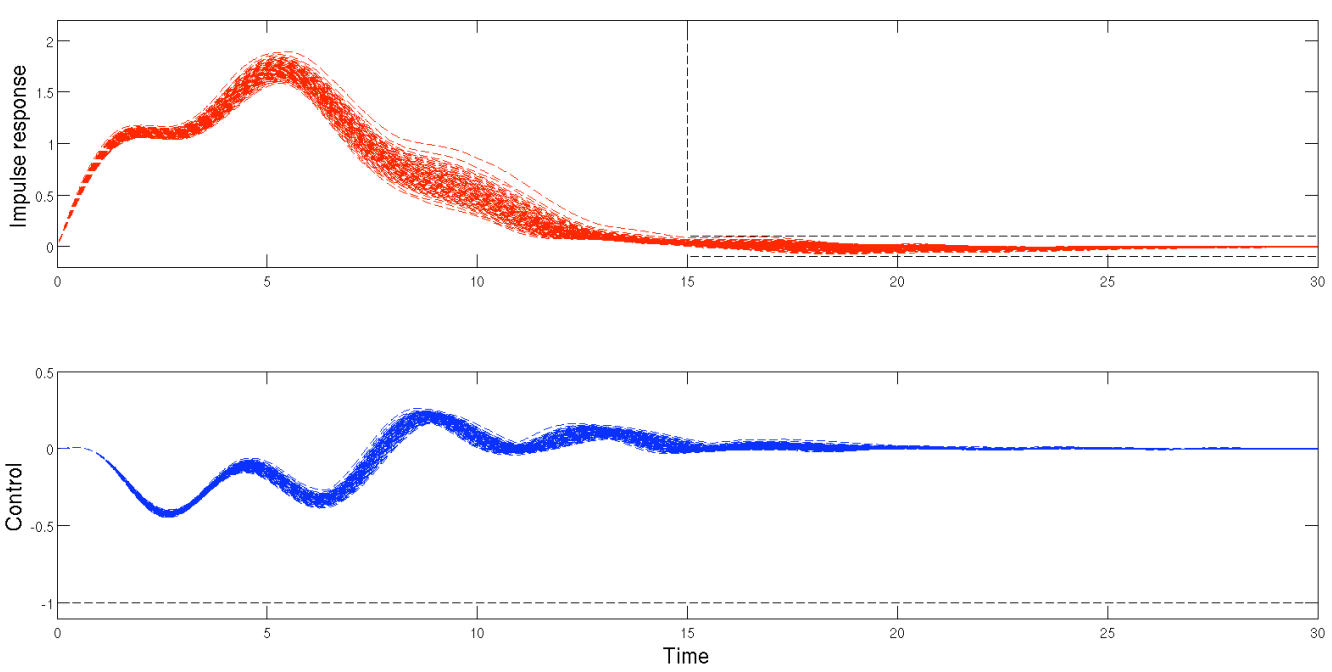

Figure 7. Percentiles $2 \%$ apart of the impulse response and control signal for $Z$.

\section{V.B. Example: Control Tuning}

In this section we search for a controller with improved robustness characteristics by applying the developments of Section IV. For this, we assume that $m_{1}, m_{2}, k, k_{n}, \tau$ and $f$ are independent, Beta distributed random variables with shape parameters, $[5,5],[5,5],[2,3.7],[6,6],[0.3,5]$, and $[0.5,1.5]$, having the support sets $[0,2],[0,2],[0.5,2],[-0.5,0.5],[\varepsilon, 0.1]$ and $[0.5,1.5]$, respectively. The ranges of variation of the parameters and the shapes of the distributions are assigned according to engineering judgment.

The spherical RIs corresponding to each individual requirement and for all controllers are provided in Table 1. According to this metric, the controller $D$ is the one with best stability and settling time characteristics while $W_{2}$ has the best figure of merit for control saturation. We will use $W_{2}$ as a baseline controller. Note that this controller does not satisfy the settling time requirement for the nominal plant. The formulation in Section IV.B led to the controller

$$
\begin{aligned}
\mathbf{A}_{c} & =\left[\begin{array}{cccc}
-2.067 & -1.049 & -0.9358 & -0.757 \\
4 & 0 & 0 & 0 \\
0 & 1 & 0 & 0 \\
0 & 0 & 0.5 & 0
\end{array}\right], \mathbf{B}_{c}=\left[\begin{array}{l}
1 \\
0 \\
0 \\
0
\end{array}\right] \\
\mathbf{C}_{c} & =\left[\begin{array}{llll}
-0.2441 & 0.1667 & 0.2567 & 0.06584
\end{array}\right] X, \mathbf{D}_{c}=0
\end{aligned}
$$

which will be labeled as controller $Z$ hereafter. A formal analysis of this controller, done using the developments in Reference [14], was performed. The stability margins attained by $Z$ are $5 \mathrm{db}$ and $31.62 \mathrm{deg}$ while the spherical reliability indices for stability, settling time and control are $\beta_{S}\left(\tilde{\boldsymbol{u}}^{1}\right)=1.746, \beta_{S}\left(\tilde{\boldsymbol{u}}^{2}\right)=0.356$ and $\beta_{S}\left(\tilde{\boldsymbol{u}}^{3}\right)=1.940$. As compared to the baseline, this controller now satisfies the design requirements for the nominal plant. As compared to all other controllers, $Z$ has a substantially better overall RI. In particular, the overall RI is more than five times larger than the one corresponding to $B_{2}$, which was the controller with best robustness characteristics. Note that the improvement in the settling time specification, which was the critical requirement, was attained by trading-off robustness in the other two specifications. 
Table 1. Spherical RIs.

\begin{tabular}{|c|c|c|c|}
\hline Controller & $\begin{array}{c}\text { Stability } \\
\beta_{S}\left(\tilde{\boldsymbol{u}}^{1}\right)\end{array}$ & $\begin{array}{c}\text { Settling time } \\
\beta_{S}\left(\tilde{\boldsymbol{u}}^{2}\right)\end{array}$ & $\begin{array}{c}\text { Control } \\
\beta_{S}\left(\tilde{\boldsymbol{u}}^{3}\right)\end{array}$ \\
\hline$A$ & 0.665 & -0.037 & 0.913 \\
\hline$B$ & 0.992 & -0.319 & 1.169 \\
\hline$C$ & 1.01 & -0.336 & 1.191 \\
\hline$D$ & 2.366 & 0.598 & $-\infty$ \\
\hline$E$ & 0.690 & -3.517 & 0.374 \\
\hline$F$ & 1.627 & 0.025 & $-\infty$ \\
\hline$H$ & 1.050 & -0.009 & 1.174 \\
\hline$W_{1}$ & 1.027 & 0.0009 & 1.152 \\
\hline$W_{2}$ & 2.147 & -0.072 & 2.287 \\
\hline$B_{1}$ & 0.497 & 0.030 & 0.005 \\
\hline$B_{2}$ & 0.852 & 0.066 & 0.236 \\
\hline$Z$ & 1.746 & 0.356 & 1.940 \\
\hline
\end{tabular}

The MS that corresponds to this controller is $\mathcal{S}_{\boldsymbol{u}}=\mathcal{S}(\mathbf{0}, 0.364)$. Therefore, the controller $Z$ satisfies the closed-loop specifications for all the members of this set. Let us consider a uniformly distributed uncertainty model having this MS as the support set. Simulations of the impulse response and control signal for the controller $B_{2}$ lead to figure 5 . Therein, $2 \%$ of the time responses are between any pair of adjacent dashed lines. The horizontal and vertical lines are used to delimit regions where the closed-loop specifications are violated. Note that the impulse response violates the requirement about $t=15 \mathrm{~s}$ with large probability while the control is at the verge of exceeding the lower limit at $t=2.5 \mathrm{~s}$. Figure 6 shows the same information for the baseline controller, $W_{2}$. As before, the settling time requirement is violated with large probability. However, considerably less actuation is now required. Figure 7 shows the responses corresponding to $Z$. Note that all requirements are satisfied, with the settling time being the critical one (i.e., the specification at the verge of being violated).

This analysis is possible because of our ability to determine the largest uncertainty set for which the closed-loop specifications are satisfied. This information cannot be obtained by any sampling method unless an infinite number of simulations are made.

\section{Concluding Remarks}

Optimization-based strategies for control analysis and tuning at the control verification stage are proposed herein. This entails dealing with complex non-linear systems having an arbitrary functional dependency on the uncertain parameters and for which stability and performance specifications are both present. The mathematical foundation enabling these developments is the ability 
to calculate sets that bound regions of satisfactory closed-loop performance. Metrics that evaluate the size of such sets are used as control verification metrics. Formulations enabling the exploration of the design space, and the improvement of the robustness characteristics of baseline controllers were proposed and exemplified. The scope and numerical requirements of the tools developed make them suitable for realistic control engineering problems.

\section{Acknowledgment}

This work was supported by the Integrated Resilient Aircraft Control Project and the Fundamental Aeronautics Project in Hypersonics from NASA.

\section{References}

${ }^{1}$ deGaston, R. and Sofonov, M., "Exact calculation of the multiloop stability margin," IEEE Transactions on Automatic Control, Vol. 33, No. 2, 1988, pp. 156-171.

${ }^{2}$ Sideris, A. and Pena, R., "Robustness Margin Calculation with Dynamic and Real Parametric Uncertainties," Proceedings of the American Control Conference, 1988, pp. 1201-1206.

${ }^{3}$ Barmish, B. R., "New Tools for Robustness Analysis," Proceedings of the 27th Conference on Decision and Control, Austin, Texas, 1988, pp. 1-6.

${ }^{4}$ Boyd, S. L., Ghaoui, L. E., Feron, E., and Balakrishnan, V., Linear matrix inequalities in systems and control theory, SIAM, Philadelphia, PA, 1994.

${ }^{5}$ Barmish, B. R. and Shcherbakov, P., "On avoiding vertexization of robustness problems: the approximate feasibility concept," IEEE Transactions on Automatic Control, Vol. 47, No. 5, 2002, pp. 819-824.

${ }^{6}$ Barmish, B. R. and Shcherbakov, P. S., "A dilation method for robustness problems with nonlinear parameter dependence," Proceedings of the American Control Conference, Denver, Colorado, 2003, pp. 3834-3839.

${ }^{7}$ Babayigit, A., Ross, B. R., and Shcherbakov, P. S., "On robust stability with nonlinear parameter dependence: some benchmark problems illustrating the dilation integral method," Proceedings of the 2004 American Control Conference, Boston, Massachusetts, 2004, pp. 2671-2673.

${ }^{8}$ Bryson, A. E. and A.Mills, R., "Linear-Quadratic-Gaussian Controllers with Specified Parameter Robustness," AIAA Journal of Guidance, Control and Dynamics, Vol. 21, No. 1, 1998, pp. 11-18.

${ }^{9}$ Tenne, D. and Singh, T., "Efficient Minimax Control Design for Prescribed Parameter Uncertainty," AIAA Journal of Guidance, Control and Dynamics, Vol. 27, No. 6, 2004, pp. 1009-1016.

${ }^{10}$ Crespo, L. G. and Kenny, S. P., "Reliability-based control design for uncertain systems," AIAA Journal of Guidance, Control, and Dynamics, Vol. 28, No. 4, 2005.

${ }^{11}$ Darligton, J., Pantelides, C., Rustem, B., and Tanyi, B., "An algorithm for constrained nonlinear optimization under uncertainty," Automatica, Vol. 35, 1999, pp. 217-228.

${ }^{12}$ Wang, Q. and Stengel, R. F., "Robust control of nonlinear systems with parametric uncertainty," Automatica, Vol. 38, 2002, pp. 1591-1599.

${ }^{13}$ Wang, Q. and Stengel, R. F., "Robust Nonlinear Flight Control of a High Performance Aircraft," IEEE Transactions on Automatic Control, Vol. 13, No. 1, 2005, pp. 15-26.

${ }^{14}$ Crespo, L. G., Kenny, S. P., and Giesy, D. P., "Figures of Merit for Control Verification," AIAA Guidance Navigation and Control Conference, Honolulu, Hawaii, USA, August 18-21 2008, p. TBD.

${ }^{15}$ Crespo, L. G., Giesy, D. P., and Kenny, S. P., "Robust Analysis and Robust Design of Uncertain Systems," AIAA Journal, Vol. 46, No. 2, 2008.

${ }^{16}$ Giesy, D. P., Crespo, L. G., and Kenny, S. P., "Approximation of Failure Probability using Conditional Sampling," 12th AIAA/ISSMO Multidisciplinary Analysis and Optimization Conference, Victoria, Canada, 10-12 September 2008, p. TBD.

${ }^{17}$ Wie, B. and Bernstein, D., "A Benchmark Problem for Robust Control Design," Proceedings of the 1990 American Control Conference, Vol. 1, San Diego, CA, USA, 1990, pp. 961-962.

${ }^{18}$ Stengel, R. F. and Morrison, C., "Robustness of Solutions to a Benchmark Control Problem," AIAA Journal of Guidance, Control and Dynamics, Vol. 15, No. 5, 1992, pp. 1060-1067.

${ }^{19}$ Wie, B., Liu, Q., and Byun, K.-W., "Robust H-infinity control synthesis method and its application to Benchmark problems," AIAA Journal of Guidance, Control, and Dynamics, Vol. 15, No. 5, 1992, pp. 1140-1148.

${ }^{20}$ Braatz, R. and Morari, M., "Robust Control for a Noncollocated Spring-Mass System," Journal of Guidance, Control and Dynamics, Vol. 15, No. 5, 1992, pp. 1103-110. 